\title{
Abrechnung Liposuktion
}

Immer wieder gibt es Probleme bei der der Abrechnung von Liposuktionen, die in medizinischer Indikation, z. B. beim Lipödem, durchgeführt werden. Da der Eingriff im GKV-Leistungskatalog nicht enthalten ist, muss in allen Fällen eine Abrechnung nach der GOÄ erfolgen. Bedauerlicherweise ist die Liquidation der Liposuktion explizit weder in der GOÄ1996 abgebildet, noch gibt es hierzu eine Stellungnahme der Bundesärztekammer.

Problematisch ist in diesem Zusammenhang der publizierte Vorschlag einer Abrechnungsmodalität, der mangels Alternativen bereits von Gerichten als Basis für deren Entscheidung herangezogen wird (Gorlas, Dtsch Arztebl 2014; 111(12): A-521/B-449/C-429). Im Wesentlichen behauptet Gorlas, dass die Liposuktion einer gesamten Extremität mit dem einmaligen Ansatz der Ziffer 2454 abgegolten ist. Begründet wird dies damit, dass die Entfernung von Fettgewebe per Liposuktion mit der Ziffer 2454 vollumfänglich abgebildet würde und daher eine analoge Abrechnung der Liposuktion ausscheide. Außerdem könne diese Ziffer nicht mehrfach je Extremität angesetzt werden, weil dies der Logik der GOÄ widerspreche. Diese Aussage ist nicht haltbar und ein Mehrfachansatz dieser Ziffer entspricht durchaus der Logik der GOÄ, wie sich leicht aus den allgemeinen Hinweisen zur Ultraschalldiagnostik ablesen lässt. Dort steht:

„Als Organe im Sinne der Leistungen nach den Nummern 410 und 420 gelten neben den anatomisch definierten Organen auch der Darm, Gelenke als Funktionseinheiten sowie Muskelgruppen, Lymphknoten und/ oder Gefäße einer Körperregion.“ (GOÄ Kapitel C VI. Ziffer 6 S. 1). Als eine Körperregion ist danach der Teil des Körpers defi- niert, welcher notfalls auch als eigener Körperabschnitt seine Funktion wahrnehmen kann. Bezüglich der unteren Extremitäten gilt als jeweils eigene Körperregion seitengetrennt definiert:

- die Leisten-Becken-Region

- der Oberschenkel bzw. die Oberschenkelmuskulatur

- das Kniegelenk

- der separate Unterschenkel

Ein derart umfassender Abrechnungsvorschlag bzw. eine Abrechnungsverhinderung wie von Gorlas im Dtsch Ärztbl 2014 mit der alleinigen und einmaligen Abrechnung der Ziffer 2454 für die Liposuktion einer gesamten Extremität muss aus folgenden Gründen abgelehnt werden:

a. Der Wortlaut der Ziffer 2454, „Entfernung von überstehendem Fettgewebe an einer Extremität" wird dem Eingriff einer Liposuktion am gesamten Bein nicht gerecht. Bei der Liposuktion wird eben nicht allein überstehendes Fettgewebe entfernt, sondern, die gesamte Oberfläche des Beines betreffend, auch nicht-überstehendes, erkranktes Lipödem-Fettgewebe abgesaugt. Eine Ziffer in der GOÄ, die diese umfängliche Behandlung abbildet, existiert nicht.

b. Über den einmaligen Ansatz der Ziffer 2454 wird der Aufwand einer Liposuktion nicht annähernd abgebildet.

c. Daher kommt hier die analoge Abrechnung nach den Regeln der $\S 6$ Abs. 2 GOÄ, zum Tragen. Nach §6, Abs. 2 GOÄ können selbständige ärztliche Leistungen, die in das Gebührenverzeichnis nicht aufgenommen sind entsprechend nach einer Art, Kosten- und Zeitaufwand gleichwertigen Leistung des Gebührenverzeichnisses berechnet werden. Vergleicht man etwa die Exzision eines Lipoms (Ziffer 2454) mit der
Liposuktion der gesamten Oberfläche eines Oberschenkels, so ist sofort klar, dass es sich um einen Eingriff handelt, der mit der chirurgischen Ziffer 2454 so nicht vergleichbar ist. Allein deshalb schon ist der Ansatz der Ziffer 2454 im Wortlaut der GOÄ für diesen extrem umfangreichen Eingriff falsch.

Die Arbeitsgemeinschaft „Liposuktion“ des Berufsverbandes der Phlebologen (BVP) gibt eine klare Empfehlung zur Abrechnung der Liposuktion. Um den Aufwand der Liposuktion (hinsichtlich der Schwierigkeit als auch zeitlich) korrekt darzustellen, wurden Areale definiert, die großen Lipomen entsprechen. Somit kann durch mehrfachen Ansatz der Ziffer 2452 A die operative Leistung der Liposuktion korrekt wiedergeben werden. In der Liquidation müssen einerseits die zeitaufwändige, gewebeschonende Tumeszenz-Lokalanästhesie, deren Verabreichung bis zu einer Stunde Zeitaufwand erfordern kann und andererseits deren zusätzliche Einwirkzeit von weiteren rund 30 Minuten OP-Raum und Personal-Ressourcen bindet. Die lymphbahnschonende Absaugung selbst unter Nutzung von besonders dünnen Absaugkanülen zieht einen weiteren zusätzlich erhöhten Zeitaufwand während der Absaugung selbst nach sich. Ein erhöhter technischer Aufwand durch Einsatz vibrierender Kanülen oder beispielsweise der Wasserstrahl-Liposuktion muss zusätzlich in der Liquidation abgebildet werden mit der Ziffer 2453A.

Zusätzliche, interstitiell angewendete hautstraffende Maßnahmen, die in ausgewählten Fällen nach Abschluss der Liposuktion z. B. an der Oberschenkelinnenseite, als separater Anschluss-Eingriff zum Einsatz kommen, müssen zusätzlich abgerechnet wer-

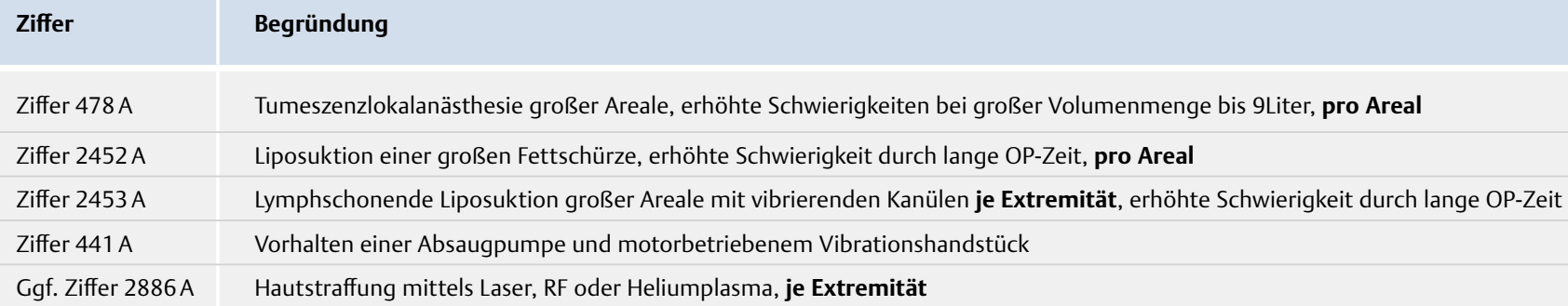

Ziffer $478 \mathrm{~A}$

Ziffer $2452 \mathrm{~A}$

Ziffer 2453 A

Ziffer $441 \mathrm{~A}$

Ggf. Ziffer 2886 A

Tumeszenzlokalanästhesie großer Areale, erhöhte Schwierigkeiten bei großer Volumenmenge bis 9Liter, pro Areal Liposuktion einer großen Fettschürze, erhöhte Schwierigkeit durch lange OP-Zeit, pro Areal Lymphschonende Liposuktion großer Areale mit vibrierenden Kanülen je Extremität, erhöhte Schwierigkeit durch lange OP-Zeit Vorhalten einer Absaugpumpe und motorbetriebenem Vibrationshandstück Hautstraffung mittels Laser, RF oder Heliumplasma, je Extremität 
den. Hierzu gehören die interstitielle Laserstraffung, interstitielle Radiofrequenz (RF)-Anwendungen und neuerdings auch die interstitielle Straffung mittels Heliumplasma. Hier empfiehlt die „Arbeitsgemeinschaft Liposuktion“ der Ansatz der Ziffer 2886 A mindestens einmal je Extremität zuzüglich der Materialkosten

Im Einzelnen empfiehlt die Arbeitsgemeinschaft „Liposuktion“ bei der Liposuktion folgende Ziffern; der Ansatz des Steigerungsfaktors erfolgt nach individuellen Gegebenheiten bis zum Höchstsatz (3,5fach):

\section{Die Aufteilung der Areale bei der Ziffer 2452 A erfolgt analog der Größe von großen Lipomen oder Fettschürzen:}

- A Gesicht: Areal 1 Wange re, Areal

2 Wange li, Areal 3 Kinn, Areal 4 Mandibula re, Areal 5 Mandibula li, Areal 6 Hals

- B Arm (Oberarm (OA), Unterarm (UA): Areal 1 UA dorsal, Areal 2 UA palmar, Areal $3+4$ OA dorsal (prox, dist), Areal
5+6 OA lateral (prox, dist), Areal $7+8$ OA ventral (prox, dist) Areal $9+10$ Axillarregion (ventral/dorsal)

- C Brust: Brust in 4 Areale einteilen (inkl. des dorsalen Anteils und des ventralen Pectoralisanteils, d.h. 4 Areale pro Brust

- D Bauch: Areal 1 Oberbauch re, Areal 2 Oberbauch li, Areal 3 Unterbauch re, Areal 4 Unterbauch li, Areal 5 Flanke re, Areal 6 Flanke li, 7 Areal Taille re, Areal 8 Taille li

- E Oberschenkel (OS): Areal 1+2 OS medial (prox/dist), Areal $3+4$ OS ventral, Areal $5+6$ OS lateral, Areal $7 \times 8$ OS dorsal

- F Unterschenkel (US): Areal $1+2$ US medial (prox/dist), Areal $2+3$ US lateral (prox/dist, Areal $4+5$ US dorsal (prox/ dist), Areal $7+8$ US ventral (prox/dist)

- G Glutealregion: gluteal re in 2 Areale einteilen, gluteal li in 2 Areale einteilen

- H Hüfte: Areal $1+2$ lateral proximal/ distal, Areal $3+4$ dorsal proximal (Übergang Taille)/distal und Areal $5+6$ ventral proximal (Übergang Unterbauch)/distal

Die Ziffer 478 A beinhaltet die Tumeszenzlokalanästhesie und wird ebenfalls in Areale unterteilt:

- Extremität: jeweils pro US, OS, UA OA 2 Areale: Zirkumferenz hälftig (medial, lateral). Für eine gesamte Extremität also 4 Areale.

- Bauch: 4 Areale

- Glutealregion: 2 Areale

- Brust: 2 Areale

- Gesicht: 1 Areal

An einer Beispielabrechnung soll aufgezeigt werden, welche Unterschiede sich aus der Anwendung der Empfehlungen des BVP gegenüber der Abrechnung nach den Empfehlungen DÄB 2014 - GOÄ Ratgeber ergeben. Das Beispiel zeigt die gleichzeitige Liposuction an beiden kompletten Oberschenkeln:

Die Abrechnung nach dem DÄB ließe nur den Ansatz der 2452 einmal je Extremität

\begin{tabular}{|c|c|c|c|c|c|c|}
\hline Ziffer & Text & Betrag $€$ & Faktor & OP-Bereich & $\begin{array}{l}\text { Areale/ } \\
\text { Extremität } \\
\text { Anzahl }\end{array}$ & Betrag $€$ \\
\hline 1 & Beratung & 4,66 & 2,3 & & & 10,718 \\
\hline 7 & Untersuchung & 9,33 & 2,3 & & & 21,459 \\
\hline 491 & $\begin{array}{l}\text { Infiltrationsanästhesie großer Areale zur Vor- } \\
\text { bereitung der Tumeszenz, Anzahl }\end{array}$ & 7,05 & 2,3 & & 4 & 64,86 \\
\hline $478 \mathrm{~A}$ & $\begin{array}{l}\text { Tumeszenzlokalanästhesie großer Areale, } \\
\text { Zeit bis zu } 60 \text { min, erhöhte Schwierigkeiten } \\
\text { bei großer Volumenmenge, je Areal }\end{array}$ & 13,41 & 2,3 & z. B. OS gesamt, beide Beine & 4 & 123,372 \\
\hline $2452 \mathrm{~A}$ & $\begin{array}{l}\text { Liposuktion einer großen Fettschürze, erhöhte } \\
\text { Schwierigkeit durch lange OP-Zeit, je Areal }\end{array}$ & 81,6 & 2,3 & $\begin{array}{l}\text { z. B. OS medial + ventral + } \\
\text { dorsal + lateral, beide Beine }\end{array}$ & 16 & 3002,88 \\
\hline $2453 \mathrm{~A}$ & $\begin{array}{l}\text { Lymphschonende Liposuktion großer Areale } \\
\text { mit vibrierenden Kanülen, erhöhte Schwierig- } \\
\text { keit durch lange OP-Zeit, je Extremität }\end{array}$ & 116,57 & 2,3 & z. B. OS gesamt, beide Beine & 2 & 536,222 \\
\hline 445 & Zuschlag ambulante Operation>1200 Punkte & 128,23 & & & & 128,23 \\
\hline 602 & Puls-Oxy & 8,86 & 2,3 & & & 20,378 \\
\hline 448 & Postoperative Überwachung & 34,97 & & & & 34,97 \\
\hline 204 & $\begin{array}{l}\text { Zirkulärer Verband mit Abpolsterung, großer } \\
\text { Aufwand }\end{array}$ & 5,54 & 2,3 & & & 12,742 \\
\hline 252 & Injektion Niedermolekulares Heparin & 2,33 & 1 & & & 2,33 \\
\hline $441 \mathrm{~A}$ & Vorhalten einer Absaugpumpe & 67,49 & & & & 67,49 \\
\hline \multirow[t]{3}{*}{ Ggf. 2886 A } & $\begin{array}{l}\text { Lipo-Laser (RF, Heliumplasma) zur Haut- } \\
\text { straffung }\end{array}$ & 161,46 & 2,3 & & 0 & 0 \\
\hline & $\begin{array}{l}\text { OP-Pack + Verbandmaterial + Absaugkanülen } \\
\text { (+ ggf. z. B. Lasersondenkosten) }\end{array}$ & 201,81 & & & & 201,81 \\
\hline & & & & & Gesamt & 4227,46 \\
\hline
\end{tabular}


zu. Alle anderen Positionen blieben unverändert! Die Gesamtrechnung beliefe sich dann auf 922,55€ und bildet damit weder den technischen Aufwand, noch den Zeitaufwand und die Schwierigkeit des Eingriffes ab.

\section{Interessenkonflikt}

Die Autoren übernehmen keine Haftung für Abrechnungen nach dieser Empfehlung.
Autorinnen/Autoren

\section{Karsten Hartmann}

Venenzentrum Freiburg,

info@venenzentrum-freiburg.de

Thomas M. Proebstle

Hautklinik, Universitätsmedizin Mainz, tom.proebstle@gmail.com

\section{Stefan Rapprich}

Hautmedizin Bad Soden,

s.rapprich@hautmedizin-badsoden.de
Bibliografie

DOI https://doi.org/10.1055/a-0889-5204

Phlebologie 2019; 48: 144-146

(c) Georg Thieme Verlag KG Stuttgart · New York ISSN 0939-978X 